\title{
Comportamento numérico e experimental de cantoneiras de aço formadas a frio
}

\author{
Ricardo Bonatto ${ }^{(1)}$, Gladimir Grigoletti ${ }^{(2)}$,Guilherme Reschke do Nascimento ${ }^{(1)}$, \\ Ignacio Iturrioz ${ }^{(3)}$, Zacarias M. Chamberlain Pravia ${ }^{(4)}$
}

\section{Resumo}

Perfís de aço formados a frio (PFF) são comumente utilizados não só em estruturas metálicas civis convencionais tais como estruturas de silos e depósitos entre outros, como também em estruturas mecânicas típicas, tais como estruturas de veículos rodoviários. A grande vantagem destes elementos é a sua versatilidade (é muito fácil fabricar perfis nas mais variadas formas de geometria de seção transversal para atender uma determinada solicitação, otimizando assim a relação resistência/peso). Por outro lado em PFF de formas não convencionais, a determinação da força resistente exige um cuidado especial em seu cálculo. $\mathrm{O}$ método tradicional de cálculo utilizado para determinar a força resistente é o Método da Largura Efetiva (MLE), simples na sua conceituação, mas trabalhoso na sua implementação. As normas mais modernas de projeto estão incorporando métodos de cálculo que possibilitam a utilização de ferramentas numéricas para determinar as forças de flambagem elástica, entre elas destacando-se o Método da Resistência Direta (MRD), proposto por Schafer e Peköz. Por outro lado, programas de elementos finitos comerciais confiáveis, que permitem avaliar diferentes tipos de não linearidades, são ferramentas que estão sendo cada vez mais utilizadas para determinar o comportamento deste tipo de perfis. Neste contexto, no presente trabalho são comparados os resultados experimentais de perfis cantoneira de diferentes comprimentos submetidos à força axial de compressão centrada com os resultados obtidos teoricamente. Para a determinação dos resultados teóricos utilizam-se o MLE, o MRD e um modelo em Elementos Finitos que considera a não linearidade física e geométrica, bem como as imperfeições geométricas iniciais dos elementos analisados. Ao final deste estudo realizam-se discussões sobre os resultados encontrados e apresentam-se conclusões sobre o comportamento dos perfis estudados como das metodologias utilizadas para sua análise.

Palavras-chave: Cantoneira de aço formada a frio. Análise numérica não linear. Imperfeições geométricas iniciais. Método da Resistência Direta.

\footnotetext{
1 Professor do FEAR/ UPF, Passo Fundo, RS, Brasil . E-mail: ricbonatto@yahoo.com.br

2 Prof. Dr. da ULBRA. E-mail: grigoletti@cpovo.net

3 Prof. Dr. do Programa de Pós-Graduação em Engenharia Mecânica da Universidade Federal do Rio Grande do Sul (PROMEC-UFRGS). Rua Sarmento Leite 425, CEP: 90050-170, Porto Alegre, RS, Brasil. Tel: +55 51 $3316-3255$. E-mail: ignacio@mecanica.ufrgs.br

4 Professor PPgEng-FEAR-UPF, Passo Fundo, RS, Brasil. E-mail: zacarias@upf.br
}

http://dx.doi.org/10.5335/rsee.v10i2.3117 


\section{Introdução}

A utilização de perfis de aço formados a frio (PFF) tem sido impulsionada nas últimas décadas, tanto pela sua facilidade de fabricação (com equipamentos simples é possível fabricar uma variada gama de seções), como pela incorporação de novas metodologias de cálculo que permitem prever a capacidade resistente de perfís com geometrias mais complexas. Até duas décadas atrás os métodos disponíveis nas normas de projeto calculavam as forças de flambagem elástica e a partir delas a capacidade resistente, utilizando expressões analíticas ajustadas (em alguns casos) com ensaios experimentais. Como consequência disso, quando não era possível o cálculo em forma analítica das forças de flambagem elástica, limitações nas relações geométricas da forma do perfil eram impostas. Com o aumento do poder de processamento dos computadores e a popularização de métodos numéricos mais robustos para o cálculo de forças de flambagem elástica, entre eles, o Método das Faixas Finitas (MFF), o Método das Faixas Finitas Restringidas (MFFr) e o Método dos Elementos Finitos (MEF), permitiu que métodos de análises mais simples pudessem ser utilizados, entre eles o Método da Resistência Direta (MRD), uma das alternativas que será explorada no presente trabalho. As sensíveis mudanças que tem ocorrido na revisão da norma de dimensionamento de PFF (a ABNT NBR 14762, 2010) e a discussão no meio acadêmico entre as potencialidades desta nova alternativa de cálculo (o MRD) mostram um campo muito fértil para pesquisas nesta área. Objetivando não buscar métodos mais fáceis e rápidos para o cálculo e projeto de PFF como aparentemente se apresenta o MRD, mas também métodos que permitam uma melhor compreensão do comportamento estrutural deste tipo de perfil e assim aproveitar melhor as possibilidades estruturais proporcionadas por formas geométricas mais complexas.

A geometria da seção transversal do perfil no qual foca o presente trabalho é a cantoneira.

Este tipo de perfil é muito utilizado, devido a sua simplicidade construtiva, na fabricação, por exemplo, de elementos estruturais secundários fazendo parte de estruturas treliçadas, elementos de ligações e contraventamentos, como exemplo pode-se citar o trabalho de Yang et. al. (2011) que discute a aplicação do perfil cantoneira na construção de torres de transmissão.

A caracterização estrutural deste tipo de perfil é um desafio, pois por trás de sua simplicidade construtiva se esconde uma alta sensibilidade às imperfeições geométricas e a um alto grau de interação entre modos locais e globais de flambagem. Como exemplo disto podem-se citar os seguintes trabalhos que estudam o comportamento de cantoneiras submetidas a forças compressão e flexo-compressão, Lui et al. 2010, 2011 e o trabalho anterior de Popovic e Hancock (1999). Em todos estes trabalhos ficam claras as dificuldades em caracterizar o comportamento deste tipo de perfis frente a estas solicitações. Neste contexto o objetivo do trabalho consiste em explorar o comportamento estrutural de perfis cantoneira utilizando também as novas ferramentas de análise mencionadas. 


\section{Metodologia}

Apresentam-se na sequência os métodos utilizados para o desenvolvimento do estudo ora apresentado.

\subsection{Análise via Método da Largura Efetiva (MLE)}

As barras submetidas à força de compressão centrada foram analisadas por meio do MLE foram analisadas utilizando-se o que prescreve a norma brasileira para o dimensionamento de perfis de aço formados a frio, a ABNT NBR 14762 (2010).

\subsection{Análise Numérica via Método da Resistência Direta (MRD)}

Nas análises através do MRD (Schafer, 2002 e Schafer e Ádány, 2006) utilizou-se o programa CUFSM (Schafer \& Peköz, 1998), para efetuar as análises de flambagem elástica local e distorcional, a formulação analítica apresentada na norma ABNT NBR 14762 (2010) para o cálculo da força axial de flambagem global elástica e para a determinação da força axial de compressão resistente a formulação contida no Anexo $\mathrm{C}$ da norma ABNT NBR 14762 (2010).

Como a cantoneira simples não apresenta um ponto de mínimo no gráfico fator de força $\times$ comprimento de meia onda utilizou-se a metodologia sugerida por Maia (2008), ou seja, para o modo local convencionou-se como o ponto de mínimo àquele correspondente a intersecção das curvas FT (modo de flexo-torção) e F (modo de flexão), e ora chamado de "mínimo convencional". Considere-se ainda que para barras com comprimento (L) menor que o comprimento de meia onda relativo ao ponto de mínimo convencional $\left(\mathrm{L}_{\text {min }}\right)$ o fator de força é o correspondente ao comprimento da barra (Figura 1).

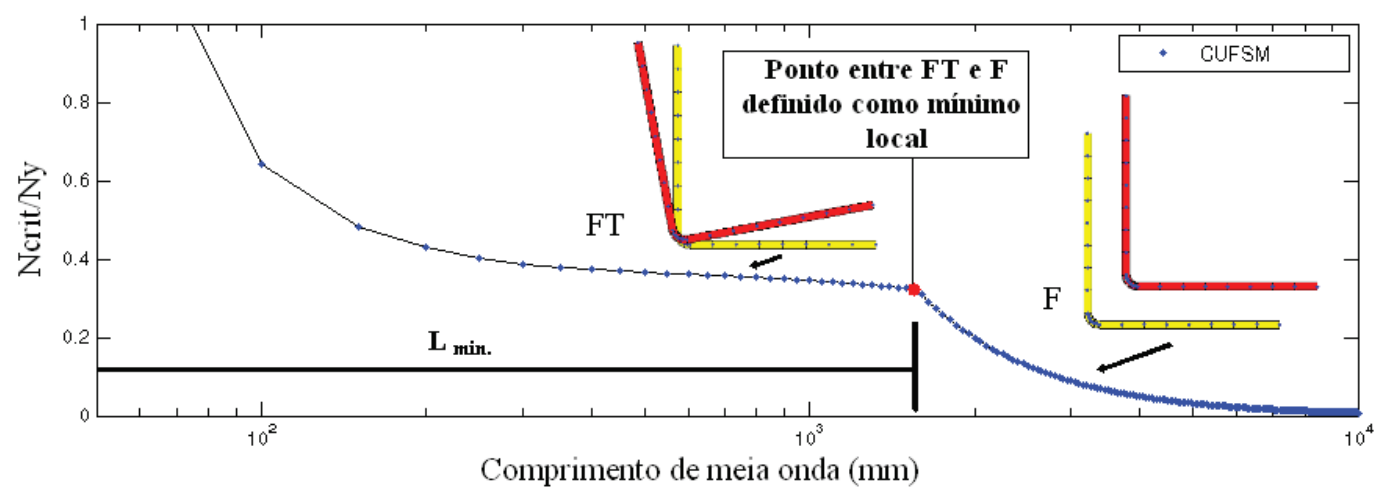

Figura 1 - Curva fator de força $\times$ comprimento de meia onda obtido via programa CUFSM para uma cantoneira simples utilizando o MFF convencional 


\subsection{Ensaios Experimentais}

\subsubsection{Caracterização do Material}

Os elementos ensaiados (cantoneiras simples) eram constituídos por aço SAE 1020 com espessura nominal de 1,06mm. Para caracterização do aço das seções formadas a frio foram selecionadas três barras e delas foram extraídos cinco corpos de prova como ilustram as Figuras 2 e 3. Os corpos-de-prova foram extraídos e ensaiados de acordo com a norma ASTM A370 (2009).

Na Figura 3 apresentam-se também as dimensões e a foto dos corpos de prova utilizados no ensaio de tração.

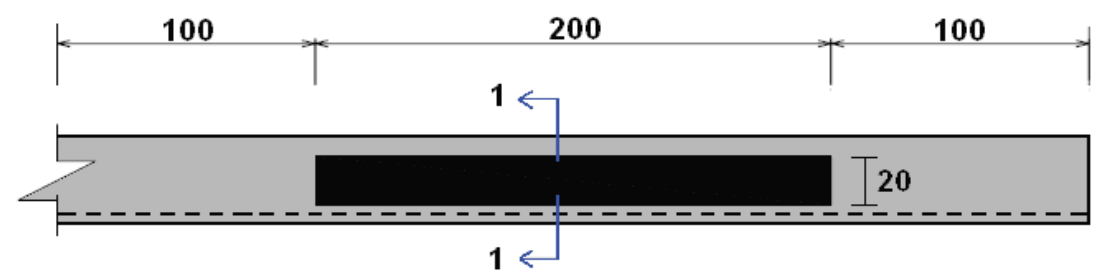

Dimensões em mm

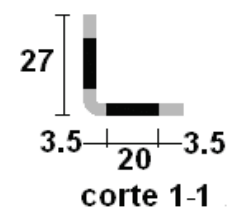

Figura 2 - Local da retirada dos corpos de prova ensaiados.
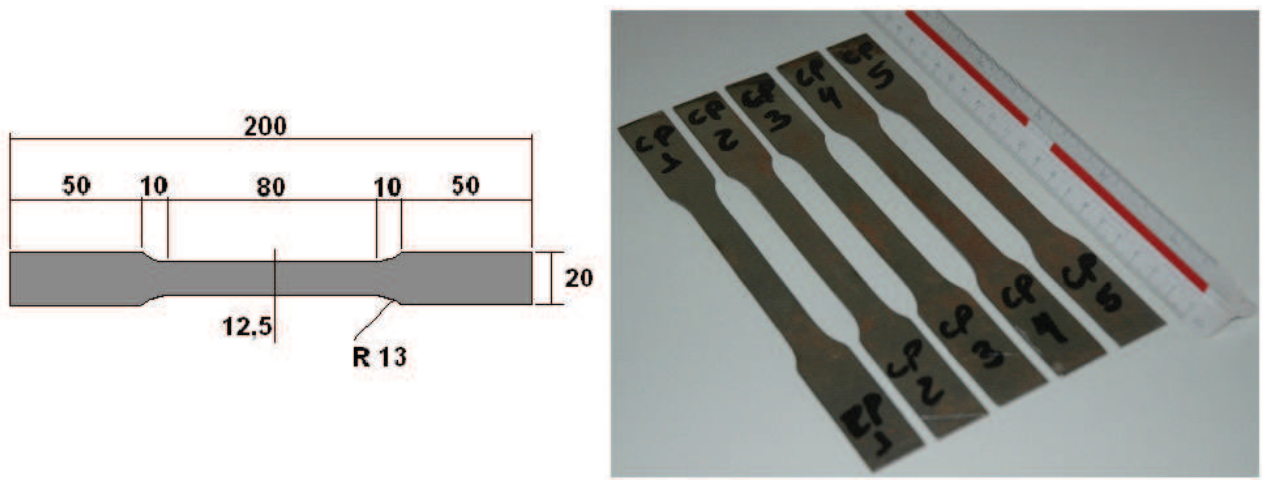

Figura 3 - Dimensões (em mm) e foto dos corpos de prova 
Os resultados relativos à resistência ao escoamento e à ruptura, bem como, o alongamento dos cinco corpos de prova apresentam-se na Tabela 1. Como o corpo de prova quatro apresentou resultados destoantes dos demais, possivelmente por problemas na aquisição de dados durante o ensaio, o mesmo foi desprezado, ficando o resultado dos quatro corpos de prova restantes (a norma ASTM A370 (2009) exige no mínimo três ensaios).

Tabela 1 - Resultados do ensaio de tração para caracterização do aço

\begin{tabular}{|c|c|c|c|c|c|c|c|c|}
\hline \multirow[b]{2}{*}{ CP № } & \multirow{2}{*}{$\begin{array}{l}\text { Área } \\
\text { Média } \\
\left(\mathrm{mm}^{2}\right)\end{array}$} & \multicolumn{3}{|c|}{ Escoamento } & \multicolumn{3}{|c|}{ Ruptura } & \multirow[b]{2}{*}{$\begin{array}{l}\text { Alongamento \% } \\
\qquad(\mathrm{L}=80 \mathrm{~mm})\end{array}$} \\
\hline & & $\begin{array}{l}\text { Força } \\
(\mathrm{kN})\end{array}$ & $\begin{array}{c}\text { Tensão } \\
\text { (MPa) }\end{array}$ & $\begin{array}{l}\text { Tensão } \\
\text { Média } \\
\text { (MPa) }\end{array}$ & $\begin{array}{c}\text { Força } \\
(\mathrm{kN})\end{array}$ & $\begin{array}{c}\text { Tensão } \\
\text { (MPa) }\end{array}$ & $\begin{array}{l}\text { Tensão } \\
\text { Média } \\
\text { (MPa) }\end{array}$ & \\
\hline 1 & 13,18 & 2,92 & 221,2 & \multirow{4}{*}{226,1} & 4,25 & 322,9 & \multirow{4}{*}{325,2} & 28,75 \\
\hline 2 & 13,13 & 2,90 & 220,9 & & 4,20 & 319,7 & & 28,00 \\
\hline 3 & 13,21 & 2,99 & 226,5 & & 4,31 & 326,1 & & 23,75 \\
\hline 5 & 13,21 & 3,11 & 235,7 & & 4,39 & 332,1 & & 30,63 \\
\hline
\end{tabular}

\subsubsection{Dispositivos Desenvolvidos para os Ensaios}

Foram confeccionados dispositivos de apoio para a fixação das extremidades dos perfis, semelhantes àqueles usados por Chodraui (2006). Estes dispositivos permitem somente giro do perfil em relação ao eixo de menor inércia, e restringindo o giro com relação ao eixo de maior inércia, a torção e o empenamento, como se pode ver na Figura 4. Cabe salientar que, como é discutido no trabalho de Young (2005), a correta fixação deste tipo de perfis, ao realizar testes experimentais, é determinante para a obtenção de bons resultados.

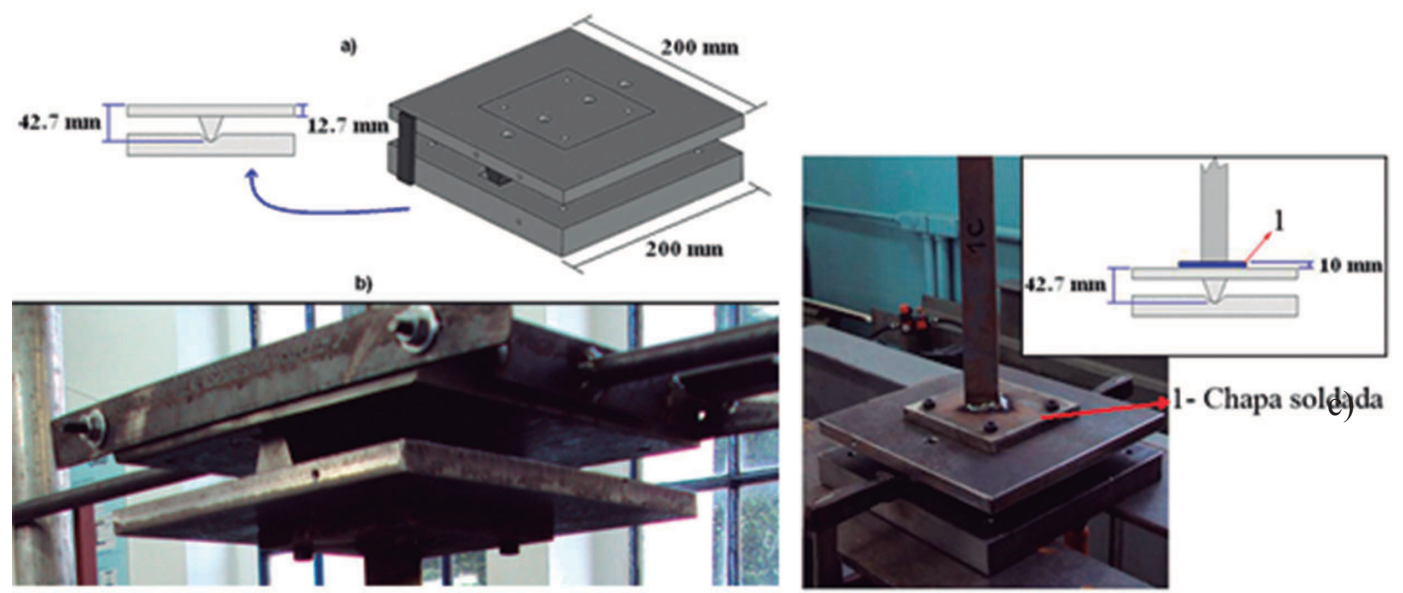

Figura 4 - Dispositivo utilizado nas extremidades: (a) Projeto utilizado para confecção com detalhe da dimensão entre a rótula e a chapa de fixação do perfil. (b) Detalhe do dispositivo e da rótula confeccionado. (c) Modelo da chapa soldada na extremidade inferior. 
Para o desenvolvimento dos ensaios experimentais foram utilizados os seguintes equipamentos:

- Como atuador de força uma bomba hidropneumática Enerpac Turbo II de capacidade máxima de $10.000 \mathrm{kgf}$.

- Para medição dos esforços utilizou-se uma célula de carga Transtec N-500 com capacidade máxima de $10.000 \mathrm{kgf}$.

- Na leitura dos dados usou-se um display digital com um micro-processador Micropan com capacidade máxima de 10.000 kgf, juntamente com o programa myPcLab versão 1.22 .

\subsection{Análise Numérica via Método dos Elementos Finitos (MEF)}

\subsubsection{Aspectos Gerais da Modelagem Numérica}

As análises numéricas foram realizadas com o uso do software de elementos finitos ANSYS versão 10.0 (2007). A simulação via MEF para efetuar as análises lineares de estabilidade e de pós-flambagem utilizadas neste estudo utilizou a metodologia adotada por Grigoletti (2008), que consiste inicialmente numa análise de flambagem elástica por autovalores (os 100 primeiros modos de flambagem são analisados). Estes modos são utilizados para modelar as imperfeições geométricas iniciais como uma combinação linear de modos de flambagem ponderadas por coeficientes empíricos gerando o campo de imperfeições geométricas iniciais. Após, incorporam-se ao modelo o campo de imperfeições geométricas iniciais determinadas anteriormente e sobre o modelo perturbado se realiza a análise não linear física e geométrica. Utilizando-se esta metodologia foi possível calcular a força e a forma de colapso da cantoneira de aço formada a frio sob compressão centrada. Detalhes sobre a fundamentação teórica deste tipo de analises assim como sobre sua implementação no MEF pode ser encontrada em Bathe (1998).

Se acontecer "softening" na curva da resposta global do modelo força versus deslocamento é necessário utilizar algoritmos mais sofisticados, um deles é o algoritmo do controle do comprimento de arco proposto por Crisfield (1981) e utilizado nos modelos numéricos deste estudo.

Note-se que a rotina criada para as análises numéricas via MEF foi calibrada através de ensaios experimentais realizados pelos autores e descritos na sequência.

\subsubsection{Elemento e Discretização Utilizada na Aplicação Desenvolvida}

Para modelar os PFF utilizou-se um elemento do tipo casca, o elemento SHELL 181, que é um elemento que possui rigidez de membrana e de flexão, apresenta quatro nós, com seis graus de liberdade cada, três de rotação (ROTx, ROTy e ROTz) e três de translação (Ux, Uy e Uz) em relação aos eixos x, y e z. Esse elemento pode considerar grandes deformações e grandes rotações. 
Já para os dispositivos das extremidades, onde são aplicadas as restrições e a força utilizou-se um elemento sólido, o elemento SOLID 45, com oito nós, sendo três graus de liberdade por nó, os três de translação (Ux, Uy e Uz).

$\mathrm{Na}$ Figura 5 pode-se visualizar o modelo discretizado com os dispositivos de extremidade e com a malha gerada.

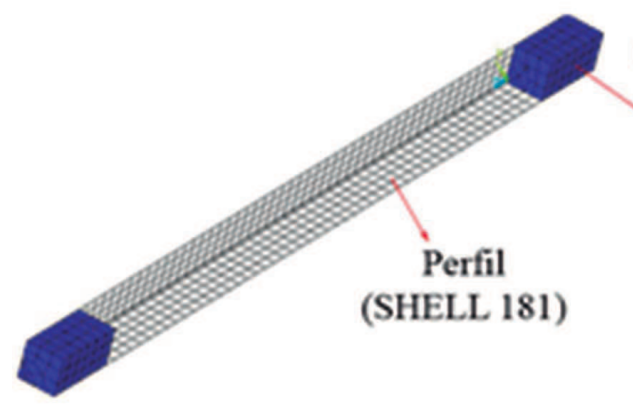

(a)

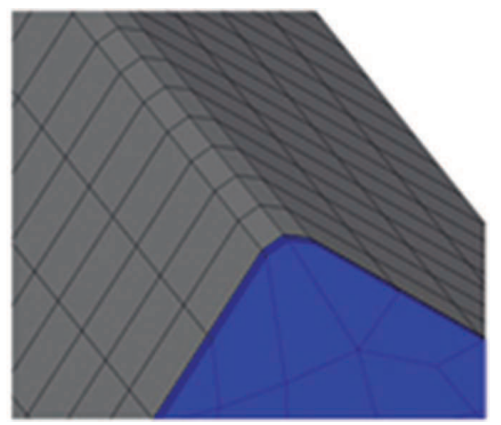

(b)

Figura 5 - (a) Modelo discretizado da cantoneira em estudo e (b) detalhe da malha no canto

Desenvolveram-se os perfis de tal forma que as suas seções transversais estão sobre os eixos x e y e o comprimento das barras fica, portanto no eixo z. Para as modelagens das seções transversais foi considerada a linha média e cantos arredondados, com raio interno igual à espessura do perfil.

Para a discretização utilizou-se uma malha de tal forma que os elementos do tipo casca fossem retangulares e os elementos do tipo sólido fossem cúbicos (sólido) com lados não ultrapassando $10 \mathrm{~mm}$, com exceção na região da dobra (canto arredondado), onde foram utilizados dois elementos como se observa na Figura 5 (b). Para definir o grau de discretização adotado foram tomados como base os resultados obtidos por Chodraui (2006).

\subsubsection{Condição de Contorno e Carregamento}

Nas cantoneiras simples, somente aquelas comparadas com os modelos experimentais, a espessura do dispositivo de extremidade foi adotada como sendo a soma da espessura da chapa do dispositivo de extremidade $(42,7 \mathrm{~mm})$ mais a espessura da chapa de topo soldada ao perfil $(10,0 \mathrm{~mm})$, sendo então adotada a espessura total de $52,7 \mathrm{~mm}$ para cada extremidade. Com isso, o comprimento entre as rótulas (Lteórica) foi calculado como o comprimento das barras (Lperfil) mais $105,4 \mathrm{~mm}$ (duas vezes $52,7 \mathrm{~mm}$ ). Detalhes sobre este particular estão ilustrados na Figura 6 para melhor compreensão. 

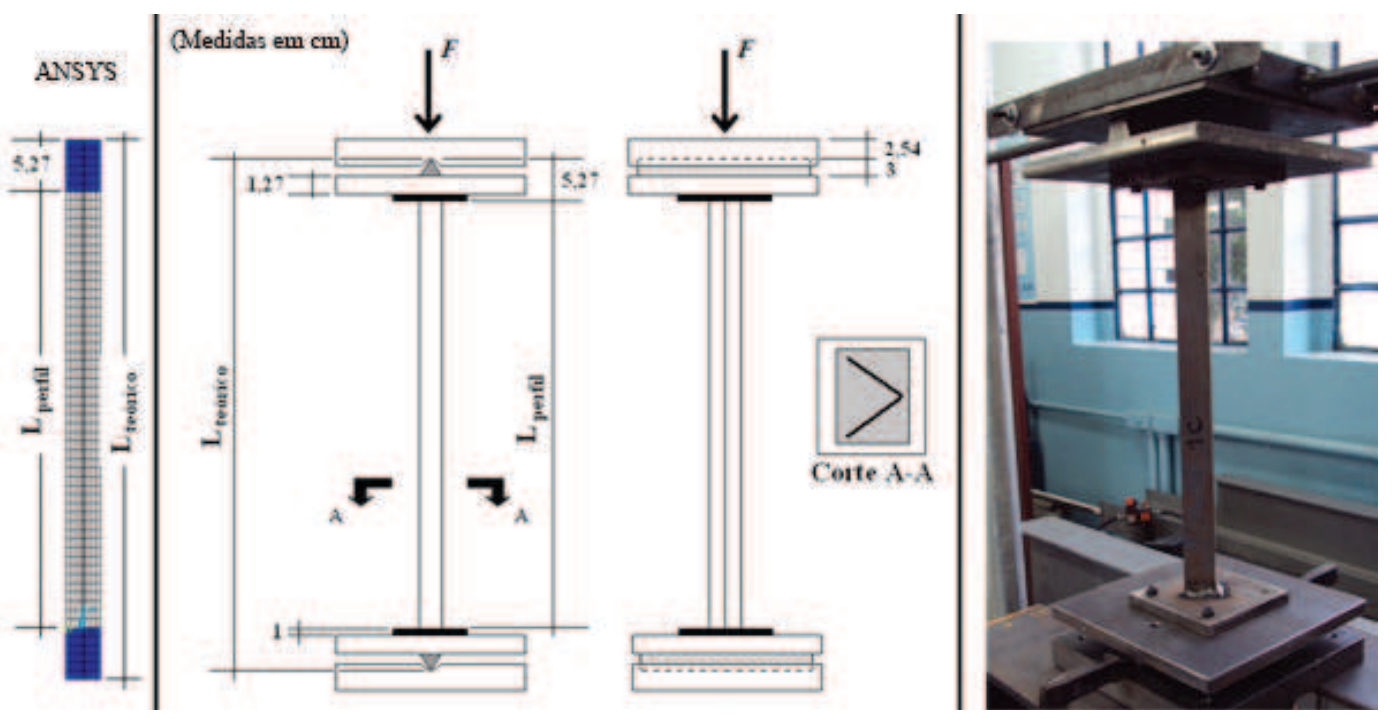

Figura 6 - Representação das cantoneiras simples com os dispositivos de extremidades e suas dimensões

Para que a análise representasse a compressão centrada, os dispositivos das extremidades foram constituídos de forma que uma linha de nós da sua malha passasse pelo centroide $(\mathrm{CG})$ da seção transversal do perfil, na direção ao eixo de menor inércia (adotado como sendo o eixo y). A Figura 7 (a) apresenta a seção transversal da cantoneira com a linha que passa pelo CG.

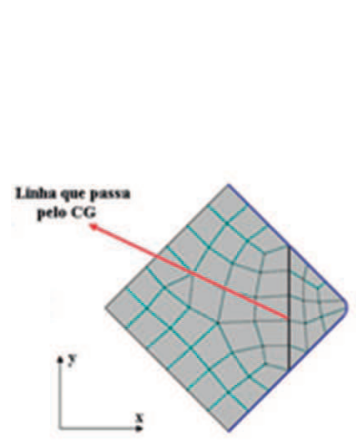

(a)

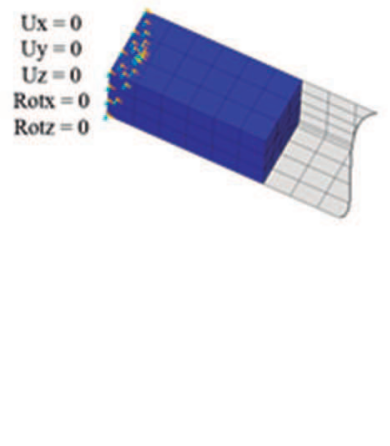

(b)

Figura 7 - (a) Seção transversal com a linha que passa pelo centroide. (b) Condições de contorno aplicadas.

A força foi aplicada na extremidade direita sobre a linha que passa pelo centroide da barra (vide Figura 7 (b)). A Tabela 2 indica as restrições adotadas nas cantoneiras estudadas. 
Tabela 2: Restrições adotadas nas extremidades da barra

\begin{tabular}{l|c}
\hline \multicolumn{1}{c|}{ Posição } & Graus de liberdade restringidos \\
\hline Nós da extremidade das chapas de topo & Ux, Uy, ROTx, ROTz \\
$\begin{array}{l}\text { Nós da linha que passam pelo CG (aplicação da força) } \\
\begin{array}{l}\text { Nós da linha que passam pelo CG (oposto à aplicação da } \\
\text { força) }\end{array}\end{array}$ & Acoplamento em Uz (Ux, Uy, ROTx, \\
Ux, Uy, Uz, ROTx, ROTz
\end{tabular}

Obs.: Eixo $z$ coincide com o eixo axial da barra

\subsubsection{Relação Constitutiva Utilizada para o Aço}

O aço foi modelado como material homogêneo e isotrópico, cujo comportamento é descrito por duas leis constitutivas, uma linear elástica (análise de estabilidade) e outra elasto-plástica tri linear (análise de pós-flambagem).

A Figura 8 apresenta o modelo constitutivo adotado para o aço adotado.

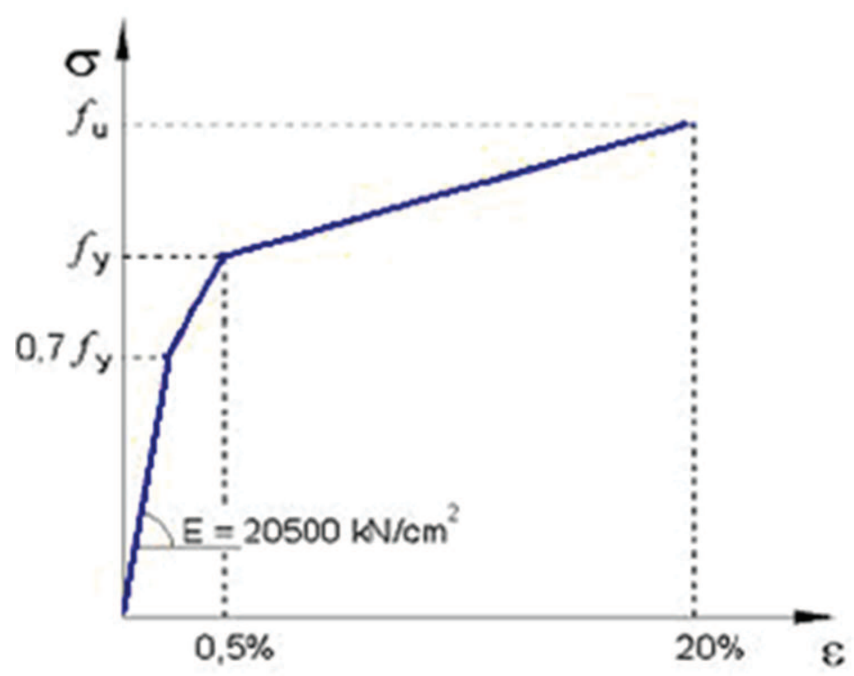

Figura 8 - Gráfico tensão-deformação para o modelo elasto-plástico trilinear adotado.

Ressalta-se que para a construção do gráfico tensão-deformação do aço, os valores adotados correspondem a valores convencionais, portanto as tensões e deformações convencionais, obtidas deste gráfico, foram convertidas para as tensões e deformações verdadeiras, uma vez que o ANSYS quando realiza a análise não linear para grandes deformações, utiliza rotinas que trabalham com valores verdadeiros de tensão e deformação (ver Equações 1 e 2). 


$$
\begin{aligned}
& \varepsilon_{t}=\ln \left(1+\varepsilon_{e}\right) \\
& \sigma_{t}=\sigma_{e}\left(1+\varepsilon_{e}\right)
\end{aligned}
$$

Onde:

$\varepsilon_{t}$ é a deformação verdadeira; $\sigma_{t}$ é a tensão verdadeira; $\varepsilon_{e}$ é a deformação convencional; $\sigma_{e}$ é a tensão convencional.

Os valores das tensões de escoamento e tensão de ruptura para a cantoneira $\mathrm{L}$ $27 \times 1,06 \mathrm{~mm}$ foram obtidos experimentalmente como indicado na seção 2.3.1 (ver Tabela $1)$.

\subsubsection{Imperfeições Geométricas Iniciais}

Um aspecto muito importante na análise numérica não linear de perfis de aço formados a frio são as imperfeições geométricas iniciais, uma vez que diferentes configurações iniciais podem mudar sensivelmente o resultado da análise. É importante ressaltar que ainda não existe um consenso, quanto à magnitude, forma e modo de como aplicar as imperfeições a serem utilizadas nos modelos numéricos.

Neste estudo utilizou-se a mesma estratégia adotada por Chodraui (2006), e Grigoletti (2008), para a inserção das imperfeições geométricas, onde inicialmente é feita uma análise de flambagem por autovalores utilizando o sistema ANSYS. Como resultado de dita análise se obteve os valores das forças críticas (autovalores) e os modos de flambagem (autovetores) associados ao modelo.

O campo de imperfeições e construído como uma combinação linear de modos de flambagem ponderados por coeficientes empíricos que regulam a participação de cada modo no campo de imperfeições.

Foram analisados os mesmos comprimentos de barra, atualizando a geometria dos perfis para as configurações onde o modo de flambagem global (flexão) produzia compressão nas bordas livres e quando o modo de flambagem global (flexão) produzia tração nas bordas livres .

A magnitude das imperfeições geométricas iniciais para o modo global foram da ordem de $L / 1500$. Mais detalhes sobre a implementação do campo de imperfeições podem ser encontrados em Grigoletti (2008).

\subsubsection{Tensões Residuais}

Chodraui (2006) observa que ainda não há um consenso na literatura de como considerar essas tensões no que diz respeito a sua distribuição e magnitude, concluindo através de ensaios que os resultados de resistência máxima também não apresentam diferenças significativas com relação a resultados de modelos sem tensões residuais.

Neste trabalho consideraram-se as tensões residuais implicitamente através do modelo elasto-plástico trilinear adotado, conforme feito por Grigoletti (2008). 


\section{Aplicação: Comportamento Estrutural da Cantoneira Simples}

\subsection{Descrição do Objeto em Estudo}

Foram estudados dois tipos diferentes de seções transversais de cantoneira simples de aço formado a frio. A Figura 9 mostra a seção transversal com a nomenclatura das dimensões das cantoneiras.

Na Tabela 3 apresentam-se as propriedades geométricas e mecânicas associadas às cantoneiras analisadas.

Foram realizadas análises para vários comprimentos das cantoneiras quando submetidas à compressão centrada.

As condições de contorno aplicadas nos extremos das barras foram detalhadas na Seção 2 (ver Figura 7). Estas condições de contorno conduzem aos coeficientes de flambagem por torção e flexão, com respeito aos eixos x e y (segundo o sistema de referência indicado na Figura $9(\mathrm{a})), \mathrm{K}_{\mathrm{x}}=\mathrm{K}_{\mathrm{t}}=0,5$ (restrição à torção e a flexão ao redor do eixo $\mathrm{x}$ ) e $\mathrm{K}_{\mathrm{y}}=1,0$ (rotação liberada ao redor do eixo $\mathrm{y}$ ).

Tabela 3 - Propriedades geométricas e mecânicas dos perfis em estudo

\begin{tabular}{c|c|c|c|c|c|c|c}
\hline \multicolumn{5}{|c|}{$\mathrm{L} 60 \times 2,38 \mathrm{~mm}$} & \multicolumn{4}{c}{$\mathrm{L} 27 \times 1,06 \mathrm{~mm}$} \\
\hline $\mathrm{b}_{\mathrm{f}}(\mathrm{mm})$ & 60,00 & $\mathrm{I}_{\mathrm{x}}\left(\mathrm{cm}^{4}\right)$ & 16,14 & $\mathrm{~b}_{\mathrm{f}}(\mathrm{mm})$ & 27,00 & $\mathrm{I}_{x}\left(\mathrm{~cm}^{4}\right)$ & 0,655 \\
$\mathrm{~b}(\mathrm{~mm})$ & 55,24 & $\mathrm{I}_{\mathrm{y}}\left(\mathrm{cm}^{4}\right)$ & 3,86 & $\mathrm{~b}(\mathrm{~mm})$ & 24,88 & $\mathrm{I}_{y}\left(\mathrm{~cm}^{4}\right)$ & 0,157 \\
$\mathrm{t}(\mathrm{mm})$ & 2,38 & $\mathrm{I}_{\mathrm{t}}\left(\mathrm{cm}^{4}\right)$ & 0,0522 & $\mathrm{t}(\mathrm{mm})$ & 1,06 & $\mathrm{I}_{\mathrm{t}}\left(\mathrm{cm}^{4}\right)$ & 0,002 \\
$\mathrm{~A}\left(\mathrm{~cm}^{2}\right)$ & 2,76 & $\mathrm{f}_{y}(\mathrm{MPa})$ & 357,0 & $\mathrm{~A}\left(\mathrm{~cm}^{2}\right)$ & 0,554 & $\mathrm{f}_{y}(\mathrm{MPa})$ & 226,0 \\
$\mathrm{x}_{o}(\mathrm{~cm})$ & 2,11 & $\mathrm{f}_{u}(\mathrm{MPa})$ & 500,0 & $\mathrm{x}_{o}(\mathrm{~cm})$ & 0,95 & $\mathrm{f}_{u}(\mathrm{MPa})$ & 325,0 \\
$\mathrm{r}_{\mathrm{o}}(\mathrm{cm})$ & 3,42 & $\mathrm{E}(\mathrm{GPa})$ & 205,0 & $\mathrm{r}_{o}(\mathrm{~cm})$ & 1,54 & $\mathrm{E}(\mathrm{GPa})$ & 205,0 \\
$\mathrm{r}_{\mathrm{m}}(\mathrm{cm})$ & 0,357 & & & $\mathrm{r}_{m}(\mathrm{~cm})$ & 0,159 & & \\
\hline
\end{tabular}

3.1.1 Descrição do Ensaio Experimental

Para a realização dos ensaios experimentais de compressão centrada nas cantoneiras simples, utilizou-se uma única dimensão de seção transversal (L 27x1,06 mm). Ensaiou-se três comprimentos diferentes de barra, $\left(\mathrm{L}_{\text {perfil }}\right): 350,450$ e $550 \mathrm{~mm}$, como mostra a Figura 9 (b). Foram feitos três corpos de prova idênticos para cada comprimento de barra ensaiado, mantendo o número mínimo de protótipos exigido pela norma ABNT NBR 14762 (2010) no seu item 11.5. 


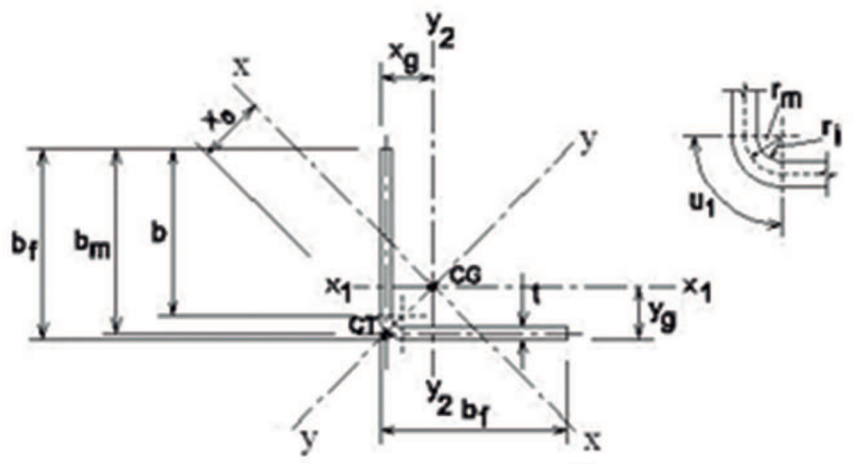

(a)

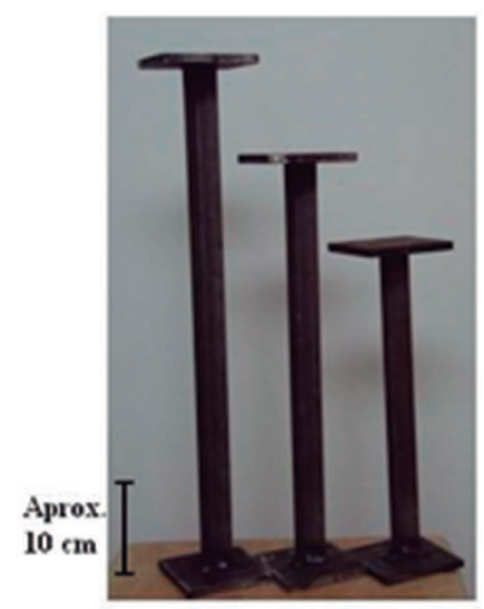

(b)

Figura 9 - (a) Seção transversal da cantoneira simples. (b) Corpos de prova ensaiados à compressão centrada.

Os resultados obtidos nos ensaios são apresentados na Tabela 3

Tabela 3 - Resultados obtidos nos ensaios experimentais ( $L 27 \times 1,06 \mathrm{~mm}$ )

\begin{tabular}{c|c|c|c}
\cline { 2 - 3 } & $\begin{array}{c}\mathrm{N}_{\text {colapso }}(\mathrm{kN}) \\
\mathrm{L}_{\text {peril }}=350 \mathrm{~mm} \\
\mathrm{~L}_{\text {teórico }}=455,4 \mathrm{~mm}\end{array}$ & $\begin{array}{c}\mathrm{N}_{\text {colapso }}(\mathrm{kN}) \\
\mathrm{L}_{\text {perfil }}=450 \mathrm{~mm} \\
\mathrm{~L}_{\text {teórico }}=555,4 \mathrm{~mm}\end{array}$ & $\begin{array}{c}\mathrm{N}_{\text {colapso }}(\mathrm{kN}) \\
\mathrm{L}_{\text {perfil }}=550 \mathrm{~mm} \\
\mathrm{~L}_{\text {teórico }}=655,4 \mathrm{~mm}\end{array}$ \\
\hline Cantoneira A & 4,90 & 5,71 & 4,42 \\
Cantoneira B & 5,13 & 4,57 & 5,40 \\
Cantoneira C & 5,20 & 4,76 & 4,36 \\
Média & 5,08 & 5,01 & 4,73 \\
\hline
\end{tabular}

Observa-se que os resultados obtidos nos ensaios cumprem com a condição prevista na norma ABNT NBR 14762 (2010), no qual, para cada comprimento analisado nenhum dos resultados apresente desvio superior a $15 \%$ do valor médio.

$\mathrm{Na}$ Figura 15 apresentam-se as configurações finais de três corpos de prova, um para cada comprimento, ilustrando que, para os dois comprimentos menores, $\mathrm{L}_{\text {perfil }}=$ 350 mm e 450 mm (Figuras 15 (a) e (b)), a configuração final foi dominada pela flambagem por flexo-torção e para o maior comprimento, $\mathrm{L}_{\text {perfil }}=550 \mathrm{~mm}$ (Figura 15 (c)), a configuração final foi dominada pela flambagem por flexão. 


\subsubsection{Cálculo Segundo a Norma NBR 14762}

A seguir são apresentados os resultados obtidos para a força resistente de cantoneiras submetidas à força de compressão centrada utilizando o procedimento da norma brasileira de perfis de aço formados a frio ABNT NBR 14762 (2010). Observa-se que no cálculo da força resistente $\left(N_{c, R d}\right)$ desconsiderou-se o coeficiente de ponderação das resistências, uma vez que a força resistente será comparado com os resultados experimentais. Então, seguindo-se o procedimento da norma, pode-se calcular a força de compressão resistente, pela Equação 3, abaixo:

$$
N_{c, R d}=\chi A_{e f} f_{y}
$$

Onde $\chi$ é o fator de redução da força axial de compressão resistente, associado à flambagem global, $A_{e f}$ é a área efetiva da seção transversal calculada segundo o MLE e que leva em conta a influência da flambagem local e, $f_{y}$ a tensão de escoamento do aço.

Na Tabela 4 são apresentados os resultados obtidos utilizando o MLE para as três configurações ensaiadas.

Tabela 4 - Cálculo do perfil L 27x1,06mm utilizando a ABNT NBR 14762 (2010) para comparação com os resultados experimentais.

\begin{tabular}{c|c|c|c|c|c}
\hline $\mathrm{L}_{\text {teórico }}(\mathrm{mm})$ & $\mathrm{L}_{\text {perfil }}(\mathrm{mm})$ & $\mathrm{A}_{\text {ef }}\left(\mathrm{mm}^{2}\right)$ & $\mathrm{N}_{\text {ey }}(\mathrm{kN})$ & $\mathrm{N}_{\text {ext }}(\mathrm{kN})$ & $\mathrm{N}_{\text {MLE }}(\mathrm{kN})$ \\
\hline 455,4 & 350 & 48,53 & 15,31 & 6,84 & 5,10 \\
555,4 & 450 & 48,59 & 10,29 & 6,81 & 5,09 \\
655,4 & 550 & 48,66 & $7,399^{\circ}$ & $6,766^{(*)}$ & 5,07 \\
\hline
\end{tabular}

$\mathrm{L}+\mathrm{F} / \mathrm{T}$ - indica que houve colapso com a interação entre a flambagem local e a flambagem por flexo-torção $\mathrm{fy}=226 \mathrm{MPa}, \mathrm{A}=55,39 \mathrm{~mm}^{2}, \mathrm{r}_{\mathrm{x}}=0,532 \mathrm{~cm} \mathrm{e}_{1}=\mathrm{K}_{\mathrm{t}}=0,5$ e $\mathrm{K}_{2}=1,0$

Os resultados obtidos para os vários comprimentos de barra das duas seções estudadas são apresentados na Figura 10 (a) e (b). Nestas figuras, $\mathrm{A}_{\mathrm{ef}}$ representa a área efetiva, $\mathrm{N}_{\mathrm{ey}}$ representa a força de flambagem elástica por flexão ao redor ao eixo de menor inércia, $\mathrm{N}_{\text {ext }}$ é a força de flambagem elástica por flexo-torção e $\mathrm{N}_{\mathrm{MLE}}$ representa a força de compressão resistente obtida utilizando o MLE, ou seja, empregando a Equação (3) anteriormente apresentada.

Nestas figuras é possível observar claramente como as diversas formas de colapso interagem. Verifica-se, por exemplo, nas duas figuras, a redução da força resistente devida a flambagem local ser dominante. Quando a área do perfil se torna toda efetiva $\left(\mathrm{A}_{\mathrm{ef}} / \mathrm{A}=1\right)$, a força resistente passa a ser dominada pela flambagem global.

$\mathrm{Na}$ Tabela 5 é apresentada a comparação entre os valores experimentais obtidos e os calculados utilizando o MLE. 


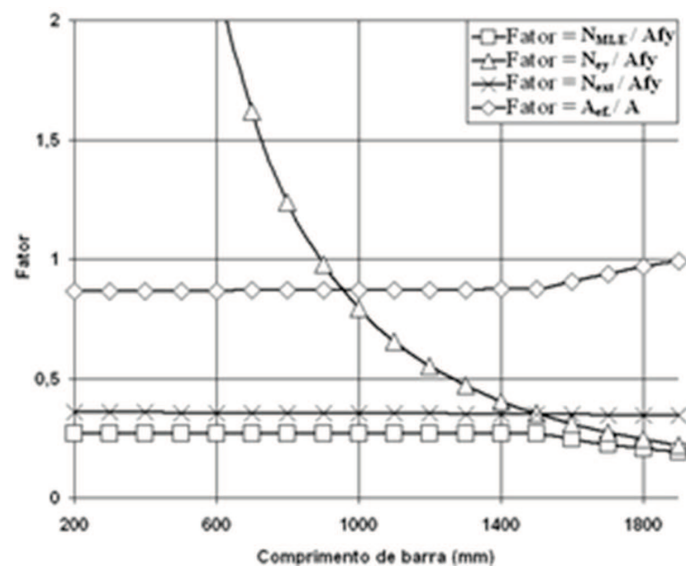

(a)

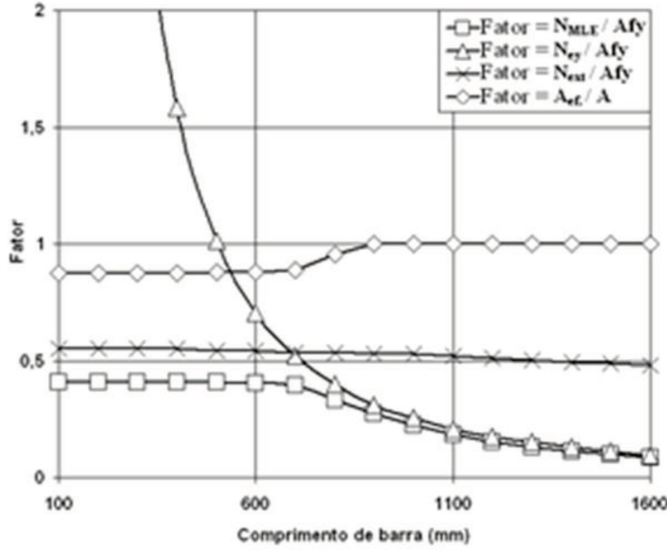

(b)

Figura 10 - Gráfico fator x comprimento de barra utilizando o MLE para as cantoneiras de seção (a) L $60 \times 2,38 \mathrm{~mm}$ e seção (b) L 27x1,06mm.

Tabela 5 - Comparação entre resultados experimentais e valores obtidos utilizando o MLE para a seção $L$ $27 \times 1,06 \mathrm{~mm}$.

\begin{tabular}{c|c|c|c|c|c|c}
\hline \multirow{2}{*}{$\mathrm{L}_{\text {teórico }}(\mathrm{mm})$} & $\mathrm{L}_{\text {perfil }}(\mathrm{mm})$ & \multicolumn{2}{|c|}{$\mathrm{NBR} 14762$} & \multicolumn{2}{|c|}{ Experimental } & \multirow{2}{*}{$\mathrm{N}_{\text {EXP }} / \mathrm{N}_{\mathrm{MLE}}$} \\
\cline { 3 - 6 } & $\mathrm{N}_{\mathrm{MLE}}(\mathrm{kN})$ & $\begin{array}{c}\text { Modo de } \\
\text { colapso }\end{array}$ & $\mathrm{N}_{\text {EXP }}(\mathrm{kN})$ & $\begin{array}{c}\text { Modo de } \\
\text { colapso }\end{array}$ & \\
\hline 455,4 & 350 & 5,10 & $\mathrm{~L}+\mathrm{F} / \mathrm{T}$ & 5,08 & $\mathrm{~F} / \mathrm{T}$ & 0,995 \\
555,4 & 450 & 5,09 & $\mathrm{~L}+\mathrm{F} / \mathrm{T}$ & 5,01 & $\mathrm{~F} / \mathrm{T}$ & 0,985 \\
655,4 & 550 & 5,07 & $\mathrm{~L}+\mathrm{F} / \mathrm{T}$ & 4,73 & $\mathrm{~F}$ & 0,932 \\
\hline
\end{tabular}

L+F/T - indica que houve colapso com a interação entre a flambagem local e a flambagem por flexo-torção

F/T - indica que o colapso ocorreu por flexo-torção, $F$ - indica que o colapso ocorreu por flexão $\mathrm{f}_{\mathrm{y}}=226 \mathrm{MPa}, \mathrm{A}=55,39 \mathrm{~mm}^{2}$ e $\mathrm{K}_{1}=\mathrm{K}_{\mathrm{t}}=0,5$ e $\mathrm{K}_{2}=1,0$

A Figura 11 (a) resume a informação apresentada na Tabela 5. 


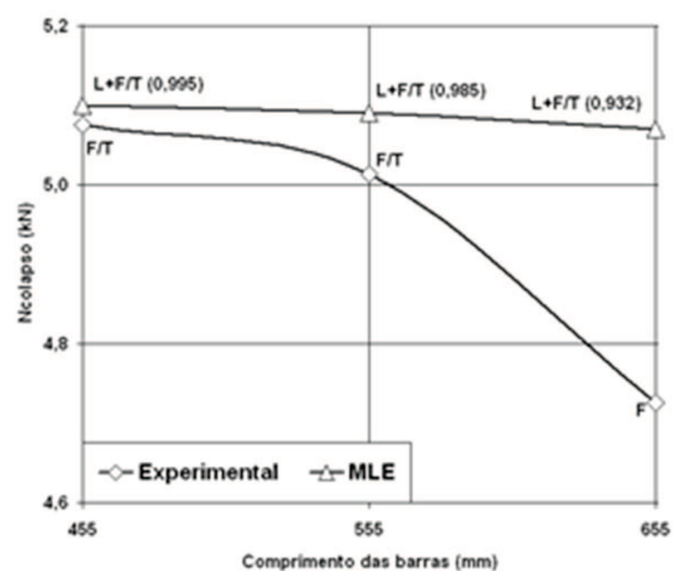

(a)

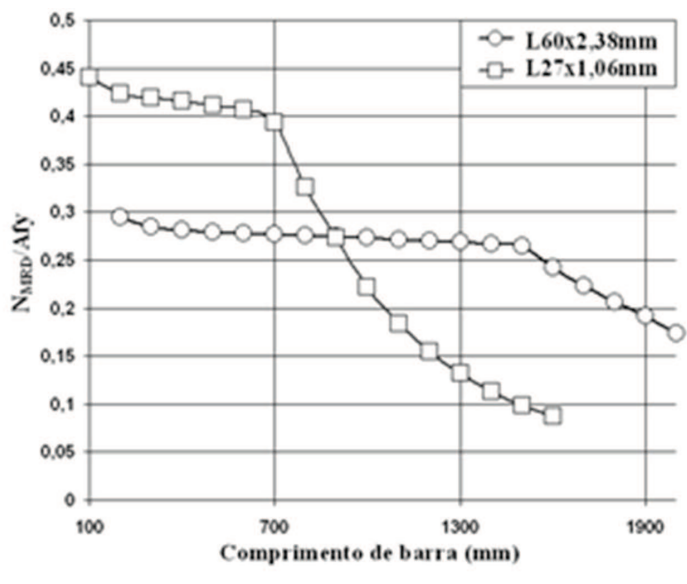

(b)

Figura 11 - (a) Resultados experimentais comparados com valores obtidos via MLE (NBR 14762) onde: $\mathrm{F}$ - flexão, $\mathrm{F} / \mathrm{T}$ - flexo/torção e $\mathrm{L}$ - local. O valor entre parênteses expressa a relação $\mathrm{N}_{\mathrm{EXP}}$. $\mathrm{N}_{\mathrm{MLE}}$ (b) Gráfico $\mathrm{N}_{\mathrm{MRD}} / \mathrm{Af}_{\mathrm{y}} \times$ comprimento de barra teórico das seções estudadas.

A partir dos resultados apresentados na Tabela 5 e Figura 11 é possível observar:

- Os dois primeiros comprimentos de barra $(455,4 \mathrm{~mm}$ e $555,4 \mathrm{~mm})$ apresentaram flambagem por flexo-torção, porém a cantoneira com comprimento de $655,4 \mathrm{~mm}$ apresentou no ensaio experimental flambagem por flexão, diferentemente do cálculo utilizando a norma ABNT NBR14762 (2010) que continua sendo flexotorção. Esta mudança de comportamento para $\mathrm{L}_{\text {teórico }}=655,4 \mathrm{~mm}$ vem justificar a diferença entre os valores experimentais e os valores teóricos (obtido via norma). O fato de esta barra colapsar por flexão, diferentemente do modo de colapso determinado via norma (flexo-torção) pode estar ligado às imperfeições geométricas iniciais provenientes do processo de fabricação destas cantoneiras.

- Na Tabela 4 os valores de força de flambagem elástica 7,39 $\mathrm{kN}^{(*)}$ e $6,36 \mathrm{kN}^{(* *)}$ (correspondentes aos modos de flexão $\left(\mathrm{N}_{\text {ey }}\right)$ e flexo-torção $\left(\mathrm{N}_{\text {ext }}\right)$ respectivamente $)$ estão bem próximos para o comprimento de barra de $655,4 \mathrm{~mm}$, deixando a barra numa região de transição entre os modos de flexo-torção e flexão. Mais uma razão que justifica a diferença na forma do colapso observado experimentalmente e a determinada teoricamente.

\subsubsection{Método da Resistência Direta (MRD)}

A descrição desta metodologia pode ser encontrada no Apêndice 1 da a norma americana para o projeto de estruturas de aço formadas a frio (NAS, 2007). Seguindo os passos especificados se apresentam a seguir os resultados obtidos ao analisar as cantoneiras estudadas. Na Figura 12 se apresentam análises de estabilidade elástica das 
seções L 60x2,38mm e L 27x1,06mm feitas com o auxílio do programa CUFSM (Schafer \& Peköz (1998) que utiliza o MFF.

Vale ressaltar que na análise de cantoneiras simples via programa CUFSM não há definido um ponto de mínimo para o modo local, portanto neste estudo optou-se em utilizar para este modo o ponto onde há mudança entre a flambagem flexo-torcional (F/T) e a flambagem flexional (F), conforme sugerido por Maia (2008).

Os fatores de força ("load factor") determinados para comprimentos de barra inferiores ao comprimento de meia onda correspondente ao mínimo estabelecido (ponto entre F/T e F da Figura 12) são aqueles correspondentes aos seus comprimentos.

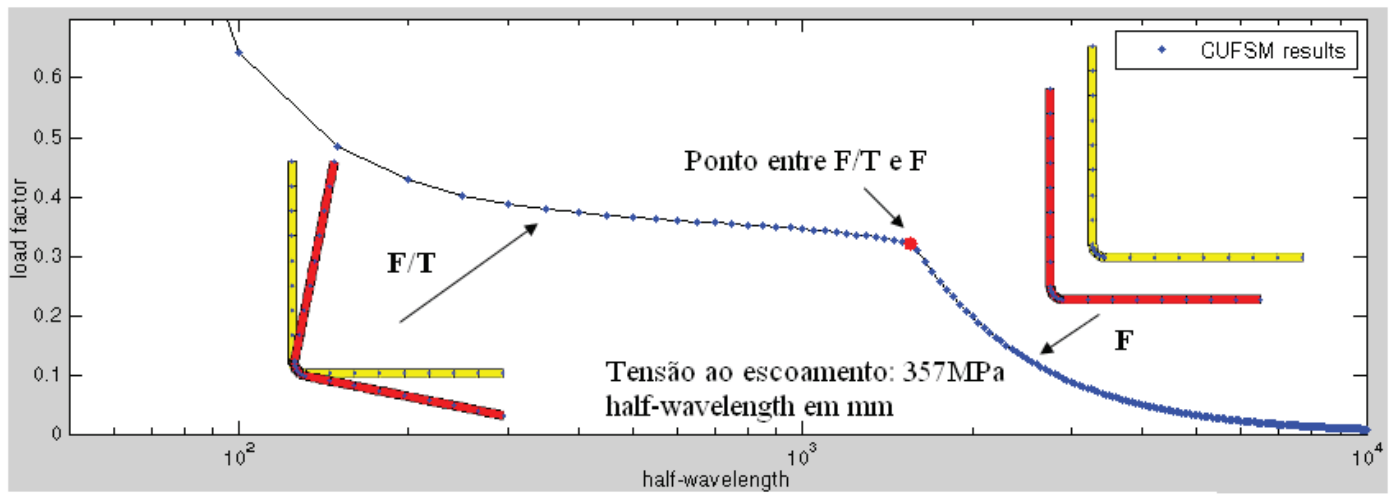

Figura 12 - Análise de estabilidade elástica via programa CUFSM da seção L 60x2,38mm

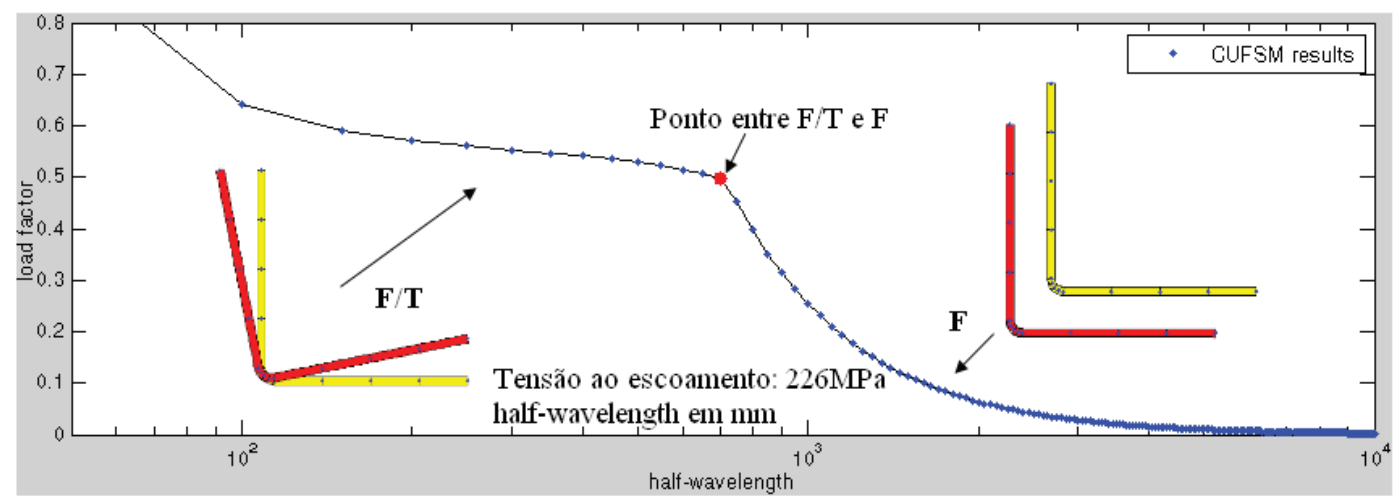

Figura 13 - Análise de estabilidade elástica via programa CUFSM da seção L 27×1,06mm

Na Figura 11 (b) apresenta-se o gráfico com os resultados obtidos em termos de $\mathrm{N}_{\mathrm{MRD}} / \mathrm{Af}_{\mathrm{y}}$ versus o comprimento teórico das barras para ambas as seções apresentadas. Observando a Figura 11 (b) é possível identificar claramente onde acontece a mudança da forma de colapso de flambagem por flexo-torção (comprimentos menores) e flambagem por flexão (maiores comprimentos). Se salienta novamente que o perfil en- 
saiado ( $\mathrm{L} 27 \times 1,06 \mathrm{~mm})$ com comprimento de $\left(\mathrm{L}_{\text {teórico }}\right) 655,4 \mathrm{~mm}$ se encontra próximo a região de transição dos modos de colapso.

Na Tabela 6está descrito a comparação dos valores obtidos segundo o MRD e os resultados experimentais.

Tabela 6 - Comparação dos resultados obtidos via MRD e obtidos experimentalmente para a seção L27x1,06mm.

\begin{tabular}{c|c|c|c}
\hline $\mathrm{L}_{\text {teórico }}(\mathrm{mm})$ & $\mathrm{N}_{\mathrm{EXP}}(\mathrm{kN})$ & $\mathrm{N}_{\text {MRD }}(\mathrm{kN})$ & $\mathrm{N}_{\text {EXP }} / \mathrm{N}_{\text {MRD }}$ \\
\hline 455,4 & $5,08(\mathrm{~F} / \mathrm{T})$ & $5,18(\mathrm{~L}+\mathrm{F} / \mathrm{T})$ & 0,98 \\
555,4 & $5,01(\mathrm{~F} / \mathrm{T})$ & $5,12(\mathrm{~L}+\mathrm{F} / \mathrm{T})$ & 0,98 \\
655,4 & $4,73(\mathrm{~F})$ & $5,06(\mathrm{~L}+\mathrm{F} / \mathrm{T})$ & 0,93 \\
\hline
\end{tabular}

$L+F / T$ - indica que houve colapso com a interação entre a flambagem local e a flambagem por flexo-torção $\mathrm{F} / \mathrm{T}$ - indica que o colapso ocorreu por flexo-torção , $\mathrm{F}$ - indica que o colapso ocorreu por flexão

$\mathrm{N}_{\mathrm{EXP}}$ - força resistente obtida experimentalmente, $\mathrm{N}_{\mathrm{MRD}}$ - força resistente obtida pelo MRD

$\mathrm{f}_{\mathrm{y}}=226 \mathrm{MPa}, \mathrm{A}=55,39 \mathrm{~mm}^{2}$ e $\mathrm{K}_{1}=\mathrm{K}_{\mathrm{t}}=0,5$ e $\mathrm{K}_{2}=1,0$

$\mathrm{Na}$ Figura 14 (a) se apresentam as forças resistentes para os comprimentos analisados utilizando o MRD e sua comparação com os resultados obtidos no ensaio experimental.

Através dos resultados apresentados acima se pode afirmar que a opção de considerar o ponto mínimo para o modo local como sendo a região de interseção entre os modos de flambagem de flexo-torção e flexão é uma boa alternativa, pois apresentou resultados satisfatórios quando comparados aos ensaios experimentais.

\subsubsection{Método dos Elementos Finitos}

Para simular o comportamento das barras analisadas neste estudo, foram criados modelos em elementos finitos com o auxílio do programa ANSYS 10 (2007).

$\mathrm{Na}$ Tabela 7 pode-se visualizar a comparação dos resultados da análise numérica via $\mathrm{MEF}$ com e os valores obtidos experimentalmente.

Nesta simulação, para o modo de flexo-torção utilizou-se a imperfeição geométrica inicial de 0,64t e para o modo de flexão adotou-se a imperfeição geométrica inicial L/1500, os mesmos valores de imperfeições iniciais propostos por Chodraui (2006).

Analisaram-se duas opções com respeito ao sentido da imperfeição geométrica inicial de flexão, ou seja, considerando o sentido da imperfeição geométrica inicial que produz tração nas bordas livres (TBL) e o sentido da imperfeição geométrica inicial que produz compressão nas bordas livres (CBL).

- A Figura 14 (b) ilustra claramente que o sentido da atualização geométrica inicial de flexão altera significativamente os resultados da força de compressão resistente. 
- Os valores encontrados através das análises numéricas utilizando o sentido da imperfeição geométrica inicial CBL mostraram-se mais próximos dos resultados experimentais do que os apresentados pelas análises com atualização da imperfeição geométrica inicial TBL, justificando-se a adoção da atualização geométrica inicial CBL.

Tabela 7 - Comparação dos resultados obtidos via MEF e obtidos experimentalmente para a seção L27X1,06mm

\begin{tabular}{|c|c|c|c|c|c|c|c|c|}
\hline \multirow{2}{*}{$\begin{array}{l}\mathrm{L}_{\text {teórico }} \\
(\mathrm{mm})\end{array}$} & \multirow{2}{*}{$\begin{array}{l}\mathrm{L}_{\text {perfil }} \\
(\mathrm{mm})\end{array}$} & \multicolumn{3}{|c|}{ MEF - Ansys } & \multicolumn{2}{|c|}{ Experimental } & \multirow{2}{*}{$\begin{array}{l}\frac{\mathbf{N}_{\text {EXP }}}{\mathbf{N}_{\text {MEF }}} \\
\text { CBL (1) }^{(1)}\end{array}$} & \multirow{2}{*}{$\begin{array}{l}\frac{\mathbf{N}_{\text {EXP }}}{\mathbf{N}_{\text {MEF }}} \\
\text { TBL }^{(2)}\end{array}$} \\
\hline & & $\begin{array}{c}\mathrm{N}_{\mathrm{MEF}}(\mathrm{kN}) \\
\mathrm{CBL}^{(1)}\end{array}$ & $\begin{array}{c}\mathrm{N}_{\mathrm{MEF}}(\mathrm{kN}) \\
\mathrm{TBL}^{(2)}\end{array}$ & $\begin{array}{l}\text { Modo de } \\
\text { falha }^{(3)}\end{array}$ & $\mathrm{N}_{\text {EXP }}(\mathrm{kN})$ & $\begin{array}{l}\text { Modo de } \\
\text { falha }^{(3)}\end{array}$ & & \\
\hline 455,4 & 350 & 4,81 & 5,26 & $\mathrm{~F} / \mathrm{T}$ & 5,08 & $\mathrm{~F} / \mathrm{T}$ & 1,06 & 0,96 \\
\hline 555,4 & 450 & 4,65 & 5,33 & $\mathrm{~F} / \mathrm{T}$ & 5,01 & $\mathrm{~F} / \mathrm{T}$ & 1,08 & 0,94 \\
\hline 655,4 & 550 & 4,34 & 5,57 & $\mathrm{~F} / \mathrm{T}$ & 4,73 & $\mathrm{~F}$ & 1,09 & 0,85 \\
\hline
\end{tabular}

(1) $\mathrm{CBL}$ - Compressão nas bordas livres, (2) TBL - Tração nas bordas livres, (3) F/T - indica que o colapso ocorreu por flexo-torção e $\mathrm{F}$ - indica que o colapso ocorreu por flexão, $\mathrm{N}_{\mathrm{ExP}}$ - força resistente obtida experimentalmente, $\mathrm{N}_{\text {MEF }}$ - força resistente obtida pelo MEF via ANSYS, $\mathrm{f}_{\mathrm{y}}=226 \mathrm{MPa} ; \mathrm{f}_{\mathrm{u}}=325 \mathrm{MPa} ; \mathrm{K}_{1}=\mathrm{K}_{\mathrm{t}}=$ 0,5 e $\mathrm{K}_{2}=1,0$. Imperfeições geométricas adotadas: fator de imperfeição para o modo de flexo-torção $=0,64 \mathrm{t}$ fator de imperfeição para o modo de flexão = L/1500.

- A partir dos resultados obtidos pode-se considerar que o modelo numérico construído no ANSYS 10 (2007) levando em conta a não linearidade física e geométrica pode ser utilizado para outros comprimentos com relativa confiabilidade, já que as diferenças entre os valores experimentais e numéricos oscilaram entre $6 \%$ e $9 \%$, que são aceitáveis.

- Ilustram-se nas Figuras 15 (a), (b) e (c) comparação das configurações finais das cantoneiras ensaiadas $\left(\mathrm{L}_{\text {térico }}=455,4 \mathrm{~mm}, 555,4 \mathrm{~mm}\right.$ e $655,4 \mathrm{~mm}$ ) com as configurações finais obtidas via MEF. Nestas figuras apresentam-se também a curva força versus deslocamento axial $U_{\mathrm{z}}$ da barra, com a indicação do ponto onde foi obtida a configuração final.

- Observe-se que os ensaios experimentais foram realizados com o controle de força o qual não permite traçar o gráfico força versus deslocamento após o colapso e que se conseguiria se o ensaio fosse realizado com o controle do deslocamento (máquina servo-controlada). 


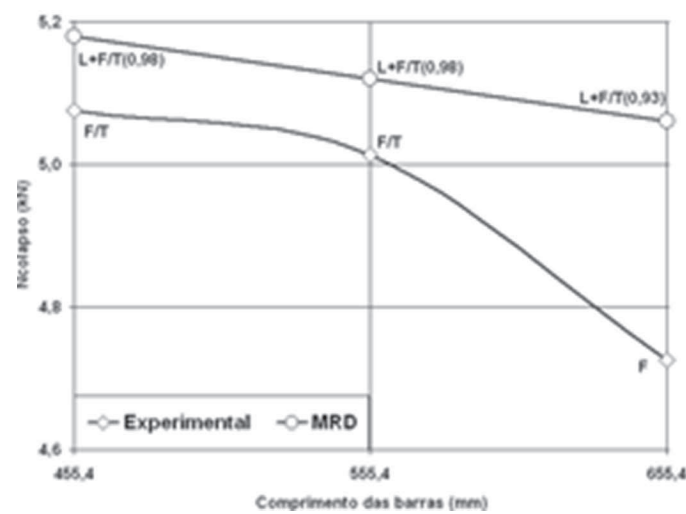

(a)

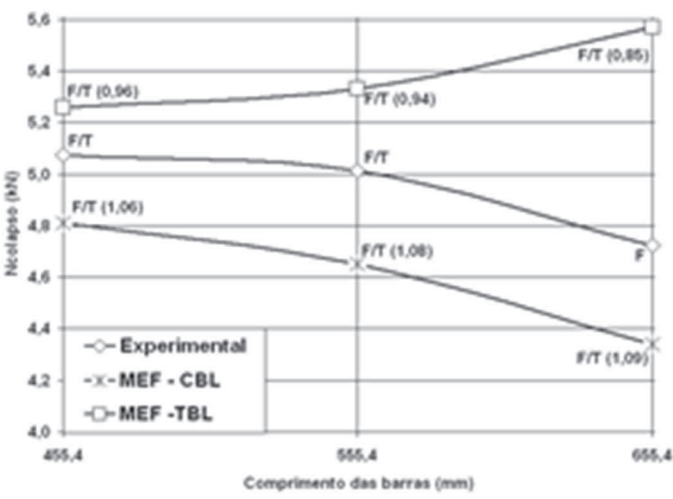

(b)

Figura 14 - (a) Resultados experimentais comparados com valores obtidos via MRD onde: F/T - flexo-torção, $\mathrm{F}$ flexão e $\mathrm{L}$ - local. $\mathrm{O}$ valor entre parênteses expressa a relação $\mathrm{N}_{\mathrm{EXP}} / \mathrm{N}_{\mathrm{MLE}} \cdot \mathrm{b}$ ) Ensaios experimentais comparados com o MEF para calibração do modelo numérico onde: $\mathrm{F}$ - flexão, $\mathrm{F} / \mathrm{T}$ - flexo/torção. $\mathrm{O}$ valor entre parênteses expressa a relação $\mathrm{N}_{\mathrm{EXP}} / \mathrm{N}_{\mathrm{MEF}}$

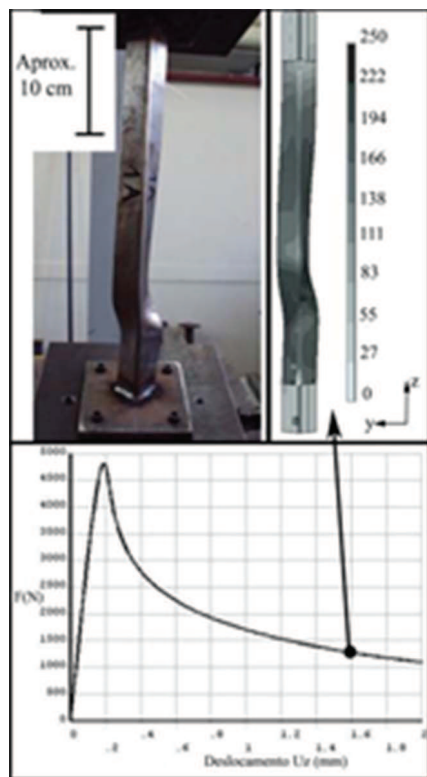

(a)

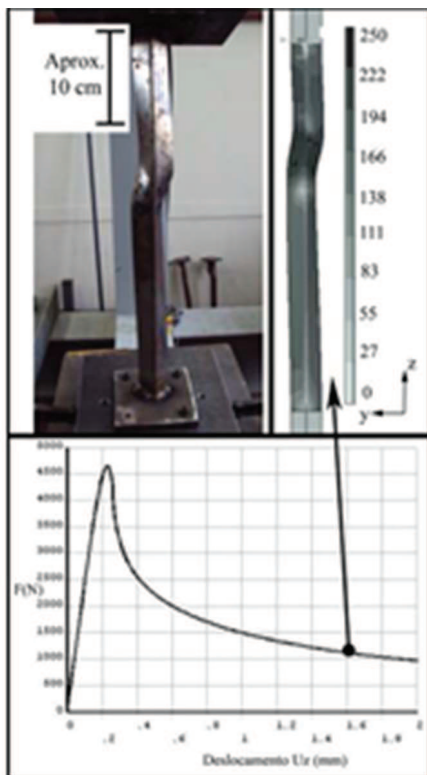

(b)

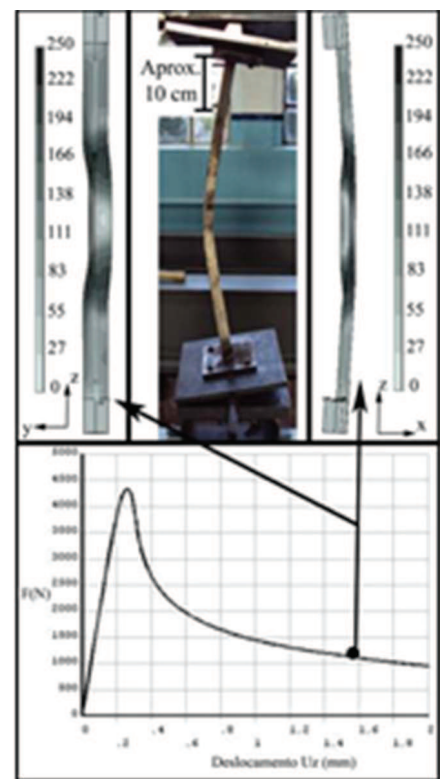

(c)

Figura 15 - Comparação das configurações finais obtidas experimentalmente e via MEF para as cantoneiras: (a) $\mathrm{L} 27 \times 1,06 \mathrm{~mm}\left(\mathrm{~L}_{\text {teórico }}=555,4 \mathrm{~mm}\right)$, (b) $\mathrm{L} 27 \times 1,06 \mathrm{~mm}\left(\mathrm{~L}_{\text {teórico }}=655,4 \mathrm{~mm}\right) \mathrm{e}$, (c) $\mathrm{L}$ $27 \times 1,06 \mathrm{~mm}\left(\mathrm{~L}_{\text {teórico }}=455,4 \mathrm{~mm}\right)$.

A seguir se apresentam nas Figuras 16 (b) e (c) gráficos resultantes do cálculo das duas seções que estão sendo analisadas para comprimentos que variam entre $\mathrm{L}_{\text {perfil }}=$ 100 até $1900 \mathrm{~mm}$. 
Para simular as imperfeições geométricas iniciais da cantoneira foi adicionada a configuração inicial um campo de deslocamentos iniciais formado pela combinação linear de um modo de flexo-torção e um de flexão, escalados por fatores empíricos.

Como fator de imperfeição do modo de flexo-torção foi utilizado os fatores $0,64 \mathrm{t}$ e 0,94 t (onde t é a espessura do perfil) e como fator de imperfeição do modo de flexão foi utilizado L/1500.

As Figuras 16 (b) e (c) ilustram o estudo paramétrico efetuado em que se variou o sentido da imperfeição geométrica de flexão, considerando tração nas bordas livres (TBL) ou compressão nas bordas livres (CBL). Na Figura 16 (a) se apresentam em forma esquemática as duas configurações mencionadas.

Observe-se que para estas análises numéricas utilizaram-se os dispositivos das extremidades com espessura igual à espessura da chapa do perfil, uma vez que as análises usando o modelo com dispositivos de extremidades com as espessuras reais das chapas mostraram que esta espessura não influi nos resultados.

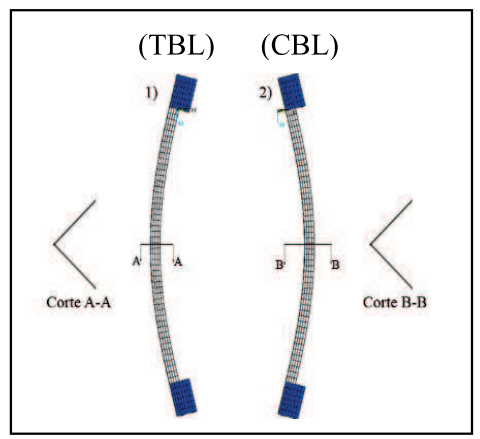

(a)

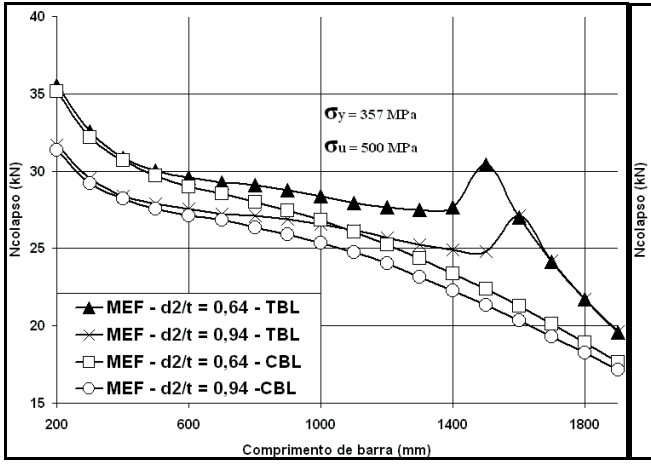

(b)

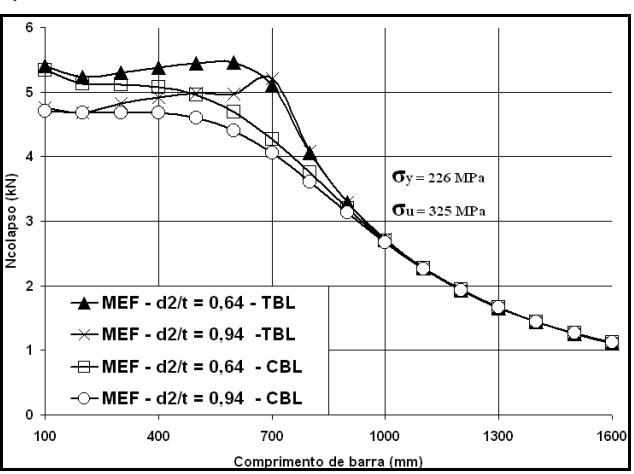

(c)

Figura 16 - (a) Esquema do sentido da imperfeição geométrica inicial TBL e CBL e gráficos da análise numérica via MEF para as seções: (b) $L 60 \times 2,38 \mathrm{~mm}$, e (c) $L 27 \times 1,06 \mathrm{~mm}$. 
Dos resultados apresentados na Figura 16 (b) e (c) pode-se observar que:

- É de fundamental importância verificar o sentido das imperfeições geométricas iniciais de flexão (casos TBL ou CBL), isto fica claro ao perceber a diferença nos resultados obtidos em ambos os casos.

- Verifica-se que ao ser utilizado a imperfeição geométrica inicial de flexão tipo TBL, não importando o valor do fator de imperfeição geométrica inicial usada para flexo-torção, ocorre um aumento acentuado da força resistente. Esse aumento da força resistente ocorre na região de transição entre os modos de flambagem dominantes (no caso, de flexo-torção para flexão).

- Quando se utiliza a imperfeição geométrica inicial do modo de flexão que produz TBL, nos comprimentos de barra onde o colapso final está associado ao modo de flexo-torção, o perfil tem que fazer um caminho inverso da atualização da geometria inicial de flexão, para que ocorra a flexo-torção (para ocorrer a F/T é necessário que a parte enrijecida tracione e as bordas livres comprimam) isso faz com que haja um incremento na força resistente.

- Quando o valor de força do modo de flambagem global elástica por flexo-torção é muito maior que o modo de flexão (barras mais longas), percebe-se que o sentido da atualização da geometria inicial de flexão (com TBL ou CBL) não influencia no resultado da força resistente. Isso também vale quando o valor de força do modo de flambagem global elástica por flexão é muito maior que o modo de flexotorção (barras mais curtas).

\subsubsection{Comparação dos Resultados entre os Métodos Analisados}

Apresentam-se nas Figuras 17 (a) e (b) os gráficos comparando as análises efetuadas utilizando diferentes métodos para as cantoneiras simples L 60x2,38mm e L $27 \times 1,06 \mathrm{~mm}$ quando submetidas à força axial de compressão centrada. 


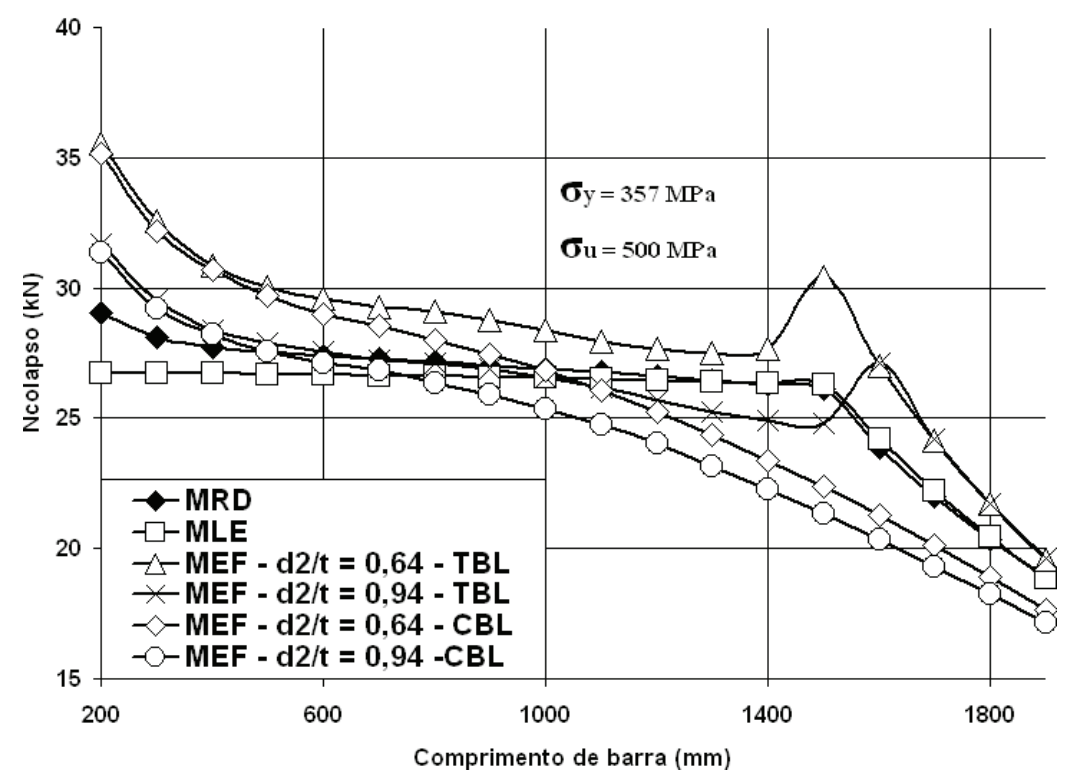

(a)

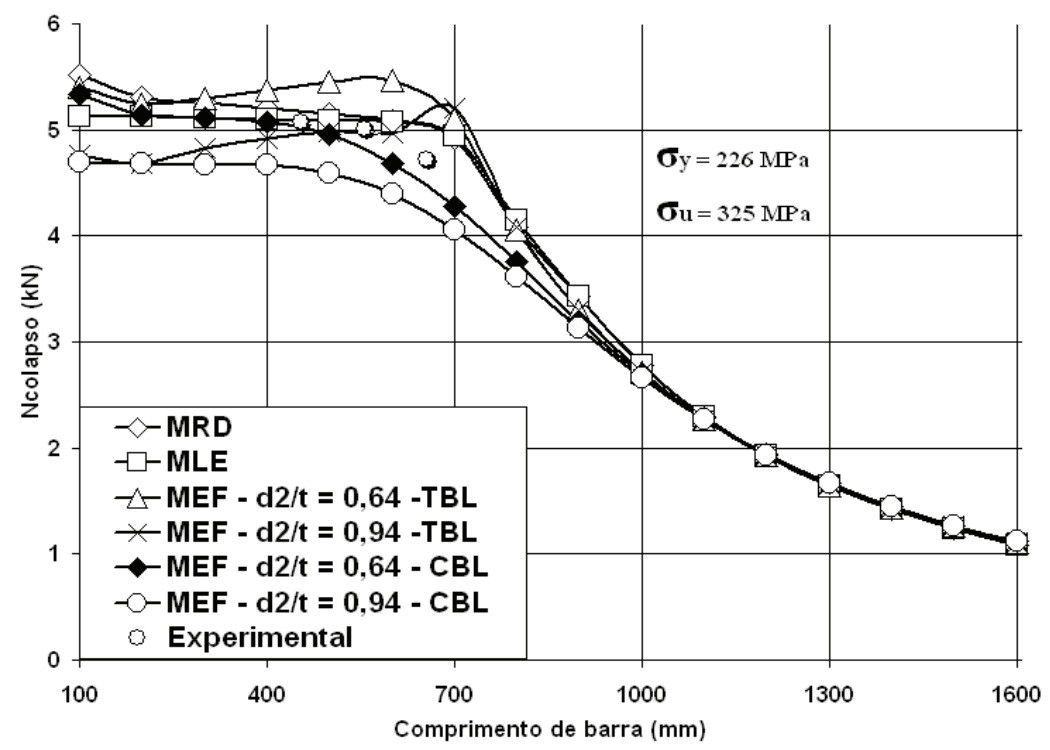

(b)

Figura 17 - Gráfico dos resultados da força resistente para todas as análises efetuadas para os perfis: (a) L 60x2,38mm e (b) L 27x1,06mm.

Realizam-se na sequência algumas observações relativas à comparação destes resultados: 
- O fator de imperfeição para o modo de flambagem de flexão utilizado para construir o campo de deslocamentos da imperfeição geométrica inicial aplicada foi de $\mathrm{L} / 1500$ em todos os casos analisados.

- Comparando os resultados obtidos para a força resistente do perfil L 60x2,38mm através do MLE e MRD (métodos da norma) com a MEF pode se perceber que até um determinado comprimento de barra $(+/-800 \mathrm{~mm})$ o valor da imperfeição geométrica inicial para o modo flexo-torcional de $0,94 \mathrm{t}$ apresenta resultados mais próximos aos do MLE e MRD, porém a partir desse comprimento de barra o valor de imperfeição geométrica inicial de flexo-torção de 0,64 t predomina com valores mais próximos aos dos métodos da norma (considerando atualização geométrica de flexão com CBL, situação mais crítica).

- Para o perfil L 27x1,06mm a imperfeição geométrica inicial de flexo-torção com valor de 0,64t apresentou valores próximos dos apresentados pelo MLE e MRD em todos os comprimentos de barras analisados para esta seção (também considerando atualização geométrica de flexão com CBL, situação mais crítica).

- Pode-se também verificar no gráfico da Figura 19 do perfil com seção L $27 \times 1,06 \mathrm{~mm}$ que a análise numérica que utiliza a imperfeição inicial de flexotorção de 0,64t e de flexão de L/1500 (com atualização geométrica com CBL) apresenta resultados muito próximos aos experimentais, validando a utilização destas imperfeições geométricas iniciais.

- Aqui cabe uma observação da importância do coeficiente de ponderação das resistências utilizada pela norma (no caso 1,20), uma vez que usando este coeficiente a força de compressão resistente de cálculo fica abaixo dos valores obtidos experimentalmente.

- A explicação para que a curva do MLE (ABNT NBR 14762, 2010), nos comprimentos de barra iniciais, não apresentar uma semelhança às curvas do MRD e MEF, pode ser dado pelo fato do MLE não considerar o cálculo do perfil como um todo e sim separadamente em placas ou também pela ocorrência de se calcular o fator de redução associado à resistência à compressão $\chi$ através da área bruta da seção transversal, não havendo uma interação com a nova área efetiva.

- Pode-se notar nos gráficos das figuras acima que o MRD comparado com o tradicional MLE são muito semelhantes. Concluindo neste caso, que a utilização do MRD para determinação da força de compressão resistente de perfis cantoneira (embora este perfil não faça parte das colunas pré-qualificadas do apêndice I da AISI (2007) e muito menos do projeto de revisão da ABNT NBR 14762 (2010) que não menciona a respeito de colunas pré-qualificadas para o MRD) é promissor, porém requerendo mais resultados para uma melhor avaliação.

- Quanto ao sentido da imperfeição geométrica inicial de flexão, comparado com os outros métodos implantados neste estudo, percebe-se que a maneira mais crítica e a favor da segurança é utilizar a imperfeição geométrica inicial de flexão considerando compressão nas bordas livres (CBL). 


\section{Conclusões}

Neste trabalho desenvolveu-se um estudo sobre cantoneiras de aço formadas a frio submetidas à compressão centrada, que tornou possível obter as seguintes conclusões:

- O Método da Resistência Direta mostrou-se de modo geral eficiente na busca da força axial de compressão resistente das cantoneiras analisadas, submetidas à força de compressão centrada, pois apresentaram respostas coerentes com as determinadas através do Método da Largura Efetiva (projeto de revisão da norma ABNT NBR 14762 (2010) e ensaios experimentais realizados).

- A utilização do programa CUFSM apresentou-se como uma ótima ferramenta para a análise de estabilidade elástica das cantoneiras simples estudadas, conduzindo a resultados semelhantes aos obtidos via MEF, adicionado ao fato de ser um software de fácil utilização e de uso livre.

- Foi importante utilizar o MEF na busca das forças de compressão resistentes das cantoneiras em estudo para diferentes comprimentos de barra, uma vez que foi possível no decorrer das análises, perceber que a consideração das imperfeições geométricas iniciais e o sentido da atualização (com TBL e CBL), principalmente para o modo de flambagem por flexão, são de extrema importância já que podem mudar significativamente a resposta final se usadas inadequadamente, uma vez que podem levar a valores contrários à segurança ou muito conservadores.

- Do exposto, pode-se afirmar que a metodologia usada neste trabalho atingiu os objetivos propostos, podendo assim mostrar a eficiência e praticidade da utilização do MRD juntamente com o MFF para determinação da força axial de compressão resistente das cantoneiras estudadas.

\section{Referências}

ABNT - Associação Brasileira de Normas Técnicas, 2010. "Dimensionamento de Estruturas de Aço Constituídas por Perfis Formados a Frio - Procedimento”, NBR 14762, Rio de Janeiro.

AISI - American Iron and Steel Institute, 2007. "NAS - North American Specification for the Design of Cold-Formed Steel Structural Members - Commentary on Appendix 1, Design of ColdFormed Steel Structural Members Using Direct Strength Method”. Washington DC.

ANSYS, Inc., 2007. “Ansys Release 10.0 Documentation”.

BATHE, K. J., 1998. "Finite Element Procedures in Engineering”, Prentice-Hall, EnglewoodCliffs.

CHODRAUI, G. M. de B., 2006. "Análise Teórica e Experimental de Perfis de Aço Formados a Frio submetidos à Compressão". Tese de Doutorado. EESC/USP, São Carlos.

CRISFIELD, M. A., 1981 "A Fast Incremental-Iterative Solution Procedure that Handles SnapThrough", Computer \& Structures, v. 13, p. 55-62.

GRIGOLETTI, G. C., 2008. "Otimização via Algoritmos Genéticos de Perfis U Formados a Frio Submetidos à Compressão Utilizando o Método Da Resistência Direta”. Tese (Doutorado em Engenharia Mecânica). Promec. Universidade Federal do Rio Grande do Sul. 
MAIA, W. F. 2008. "Sobre a Estabilidade de Cantoneiras de Aço Formadas a Frio Submetidas à Compressão”. Dissertação (Mestrado) - Escola de Engenharia de São Carlos, Universidade de São Paulo.

LIU, Y., Hui, L., (2010) "Finite Element study of steel single angle beam-columns". Engineering Structures, Vol. 32, pp. 2087-2095

LIU, Y., Chantel, S. "Experimental study of steel single unequal-leg angles under eccentric

Compression”. Journal of Constructional Steel Research 67 (2011), pp.919-928.

POPOVIC, D., Hancock, G. J., and Rasmussen, K. J. R. (1999). "Axial compression tests of coldformed angles”. Journal of Structural Engineering, 125(5), pp. 515-523.

SCHAFER, B. W.; Peköz, T. 1998. "Computational Modeling of Cold-Formed Steel: Characterizing Geometric Imperfections and Residual Stresses”. Journal of Constructional Steel Research, v. 47 , p. 193-210, January.

SCHAFER, B. W., 2002. "Design Manual for Direct Strength Method of Cold-Formed Steel Design”. Draft for American and Iron Steel Institute - Committee on Specifications, January 7, 2002.

SCHAFER, B. W., Ádány, S., 2006. "Buckling Analysis of Cold-Formed Steel Members Using CUFSM: Conventional and Constrained Finite Strip Methods", Proceeding of the Eighteenth International Specialty Conference on Cold-Formed Steel Structures, Orlando, Florida, October.

YOUNG, B. (2005). "Experimental investigation of cold-formed steel lipped angle concentrically loaded compression members”. Journal of Structural Engineering, 131(9), pp. 1390-1396.

YANG, F., Han, J., Yang, J., Li, Z. (2011) "Study on the buckling behavior of cold-formed angles in transmission towers". International Journal of Steel Structures, Vol. 11, Issue 4, p. 495-508. 


\title{
Numerical and experimental behavior of cold-formed angle sections
}

\begin{abstract}
Cold-formed sections are not only applied to steel structures in buildings, other applications are bunks, silos, and also mass transport vehicles. The main advantage of those sections is its formability, i.e., the easy way to develop any geometrical section. However this advantage demands a careful estimation of the squash load. The common method used is the effective width (EWM), to get properties of the section and ultimate load, easy as a concept, difficult to implement numerically. Modern standar$\mathrm{ds}$ for the project are incorporating new numerical methods to determinate the elastic buckling force, among them the Direct Strength Method (DSM). Finite Element commercial software's, also, allow develop non-linear behavior of several kinds, which result in better limits loads. In lieu of that facts, in the present work are developed numerical and experimental studies for different lengths of steel angle section subjected to compression, and both results are analyzed and commented. For numerical estimation of buckling load was used the DSM and the EWM, and also a finite element analysis with include physical and geometrical nonlinearity and the imperfections of the elements experimental studied. At the end of this study are held discussions about the results and present conclusions about the behavior of the section studied as well as the methodologies used for their analysis.
\end{abstract}

Keywords: Cold-formed angle. Nonlinear numerical analysis. Initial geometric imperfections. Direct Strength Method.

\section{Introduction}

This work studied the cold-formed angle section widely used due to its constructive simplicity, in manufacturing, for example, of secondary structural elements as part of truss structures for roofs, elements of connections and bracings. Structural characterization of this type of open section is a challenge, because behind its constructive simplicity hides a high sensitivity to geometrical imperfections and great interaction between local and global buckling modes. The objective of the present work is to study the behavior of this type of section subjected to compressive loads, using the classical methodology suggested by several standards, employing the direct strength method, which combines numerical results of buckling elastic and semi empirical resistance curves and non-linear finite element solutions. To compare the results obtained were also conducted experimental tests. 


\section{Methodology}

The methods used for the development of this work were:

Analysis by the effective width method (EWM) :The elements subjected to axial compression were analyzed by the Brazilian Standard, through the effective width method, ABNT NBR 14762:2010.

Analysis by the Direct Stregnth Method (DSM): The analysis through DSM (Schafer (2002) and Schafer, Ádány, (2006)) the free software CUFSM (Schafer \& Peköz, 1998), for the elastic buckling analysis with the finite strip method. The collapse load use the formulation in the annex C from ABNT NBR 14762:2010. The global flexure mode is the Euler formulation, the local mode use the criteria adopted by Maia(2008), which use as local mode the transition point between the global modes of flexure and flexure and torsion obtained from CUFSM, these behavior is exposed in the Figure 1.

Non-linear Finite Element Model: The numerical analysis via MEF adopted in this study was implemented in ANSYS 10 (2007). This analysis includes the geometrical and material nonlinearities. Introducing in the model initial imperfections obtained as a combination of parameters. Details about the construction of the model can be found at Grigoletti (2008). The model and the particularities relating to boundary conditions applied can be found in Figures 6 and 7 .

Experimental Tests: Simple tension coupon tests were carried out to characterize the behavior of the material. Coupons and where were extracted are detailed in Figure 2 . The tests of angles were carried out using a cross-section ( $\mathrm{L} 27 \times 1.06 \mathrm{~mm})$, with three different bar lengths, $\left(\mathrm{L}_{\text {perfil }}\right)$ of 350, 450 and $550 \mathrm{~mm}$, as shown in Figure $9 \mathrm{~b}$. Three identical specimens for each test bar length. Was developed a special device for the load application to maintain its application centrally the center of gravity of the section as shown in figures 6 and 9.

\section{Results}

Here were studied two different types of cross sections of single cold-formed steel angles. In table 3 are the geometric and mechanical properties associated with those angles. Analyses were carried out for various lengths when subjected to compression centered. The results obtained by applying all methods before described together with the experimental results are presented in figures 18. $a$ and $b$. 


\section{Conclusions}

In this work a study on cold-formed steel angles was developed. Some aspects need to be highlighted as possible conclusions:

- The Direct Strength Method showed efficiency for the search of the buckling axial load of the angles analyzed.

- It was essential to use the FEM in search of buckling loads in compression for different lengths of the bar, since it was possible in the analysis realize the consideration of the initial geometric imperfections and sensitive of its inclusion. 